\title{
Off-label Prescriptions: how to Identify Them, Frame Them, Announce Them and Monitor Them in Practice?
}

\author{
2 Laboratoire Sanofi, Paris, France \\ 3 Haute Autorité de Santé, Saint-Denis la Plaine, France \\ 4 Centre hospitalier de Bordeaux, Bordeaux, France \\ 5 Orphandev, Centre hospitalier de Marseille, Marseille, France \\ 6 Centre hospitalier Lariboisière, Paris, France \\ 7 Pharmacologie, Faculté de Médecine, Lille, France \\ 8 Les entreprises du médicament (LEEM), Paris, France \\ 9 Haute Autorité de Santé, Saint-Denis la Plaine, France \\ 10 Laboratoire GlaxoSmithKline, Marly-le-Roi, France \\ 11 Laboratoire Novartis Pharma, Rueil-Malmaison, France \\ 12 Laboratoire Lundbeck, Issy-les-Moulineaux, France \\ 13 Centre hospitalier de Nantes, Nantes, France \\ 14 Cabinet de Médecine générale, Saint-Épain, France \\ 15 Pharmacie Hôpital Robert Debré, Paris, France \\ 16 Centre hospitalier Saint Antoine, Paris, France \\ 17 Laboratoire Lilly France, Suresnes, France \\ 18 Laboratoire Celgene, Paris, France
}

Claire Le Jeunne ${ }^{1}$, Nathalie Billon ${ }^{2}$, Anne Dandon ${ }^{3}$ and participants of round table $N^{\circ} 3$ of Giens XXVIII: Driss Berdai ${ }^{4}$, Yolande Adgibi ${ }^{5}$, Jean-François Bergmann ${ }^{6}$, Régis Bordet ${ }^{7}$, Anne Carpentier ${ }^{8}$, Emmanuelle Cohn ${ }^{9}$, Soizic Courcier ${ }^{10}$, Danièle Girault ${ }^{11}$, Sylvia Goni ${ }^{12}$, Pascale Jolliet ${ }^{13}$, François Liard ${ }^{14}$, Sonia Prot-Labarthe ${ }^{15}$, Tabassome Simon ${ }^{16}$, Christine Vernotte ${ }^{17}$ and Jérémie Westerloppe ${ }^{18 \dagger}$

1 Service de Médecine interne, Assitance Publique - Hôpitaux de Paris, Hôtel Dieu, Paris, France

Text received April 12 ${ }^{\text {th }}, 2013$; accepted May, $7^{\text {th }} 2013$

\section{Keywords:}

MA;

off-MA;

conditonal MA; temporary treatment protocol; regulation; temporary use recommendations

\begin{abstract}
Following the Mediator crisis and the passage of the Health and Safety Law of December 2011, off-label prescriptions are a real concern shared by all those involved in healthcare system. Off-label, in the strictest sense of the term, is defined as all prescriptions that do not correspond to the summary of product characteristics (SPC), particularly those that fail to comply with the indications and dosage regimens defined by the marketing authorization (MA) for clear safety reasons. There are various rasons for off-label prescriptions, both conscious and unconscious. They are intended to respond to unmet medical needs, the needs of poorly studied populations or not studied at all in trials, but in relation to whom it is reasonable to extrapolate that MA would be given (common-sense prescriptions) and, additionally, to urgent public health needs (such as baclofen, pregnant women, and HIV drugs). All these prescriptions would deserve to be studied for a potential MA. However, there are off-label prescriptions that need to be restricted or even penalized in the case of compassionate prescriptions or unjustified prescriptions or prescriptions not based on any scientific grounds.

Off-label prescriptions are not easy to track down because if the prescriber has to write "off-label" on his prescription, then clearly, in practice, he will only do so in exceptional cases. Neither the pharmacists who dispense the drug nor the Social Security that reimburses it, have access to the diagnosis (or targeted indication). Thus, in order to identify the off-label prescription, we must be able to cross reference the available databases (such as pharmacovigilance database, medicalized information
\end{abstract}

$\dagger$ Articles, analyzes and proposals from the Giens workshops are those of the authors and do not prejudice the position of their parent organization. 
system program [programme de médicalisation des systèmes d'information, PMSI], hospital drug formularies, general sample of beneficiaries [échantillon généraliste de bénéficiaires, EGB] or national inter-regional Health Insurance Information System [système national d'informations inter-régions d'Assurance maladie, SNIIRAM], sales data, and data from market surveys). The shared computerized patient file may resolve this problem. The temporary use recommendation (TUR) proposed by the Drug Safety Law will only partially deal with this problem for recently marketed molecules.

This temporary and exceptional mechanism will authorize a recognized off-label prescription, which may be reimbursed and monitored for 3 years. These TURs will only concern a small portion of "off-label" drugs having yet a positive risk/benefit ratio (conditional MA) but this is far from matching with majority of off-label prescriptions. As such, and in order to improve the use of drugs, it is important to propose a control system for all "off-label" prescriptions with a dedicated committee: the "off-label" committee which would determine the frame of the "off-label" prescriptions.

Abbreviations: see end of article.

\section{Introduction}

In 2010, the Mediator crisis highlighted the risks by using the drug out of the scope of its marketing organization (MA). Such a use is a widespread practice and has become a concern shared by all the stakeholders involved in the healthcare system. During the Assises du médicament (drug conference), a group has been specifically assigned for providing some recommendations on the offlabel prescriptions. ${ }^{[1]}$

The recommendations of this group were:

1. To track and to monitor the "off-label" prescriptions.

2. To track the risks of "off-label" prescriptions from clinical trials and consequently to reinforce the public research.

3. To improve the reliability of the "off-label" prescription recommendations issued by the health authorities (such as High Health Authority [Haute autorité de santé, HAS], National Insitute against Cancer [Institut national contre le cancer, INCA]), and French Agency for the Safety and Health Products [Agence française de sécurité sanitaire des produits de santé, Afssaps]).

4. To report more frequently the unjustified "off-label" prescriptions, particularly those that are safety concerns.

5. To reinforce the exemption mechanisms: temporary use (autorisation temporaire d'utilisation, ATU), temporary treatment protocol (protocole thérapeutique temporaire, PTT) or "article $56^{\text {"[2] }}$ by strongly monitoring the patient follow-up and by scheduling the end of such systems.

6. To gradually extend the article 56 model to all off-label prescription recommendations issued by the health authorities.

7. To mobilize all the Health Authorities like the HAS, the National Security Agency of Medicines and Health Products (Agence nationale de sécurité du médicament et des produits de santé, ANSM), the INCA and the Economic Committee for Health Products (Comité économique des produits de santé, CEPS) and to ensure their coordination under the responsibility of the Health Ministry.

8. To inform patients and healthcare professionals.

9. To give greater responsibility to in the healthcare system stakeholders.
These mostly common-sense recommendations were the basis of the health and safety law of December 2011, which specifies in the Public health code (Code de la santé publique, CSP) the acceptable conditions for off-label prescriptions and the creation of a new control mechanism for medically justified off-label prescriptions: temporary use recommendations decided and centralized by the ANSM. $^{[3]}$

Currently, all the needed regulatory texts for the implementation of this mechanism have been published. ${ }^{[4-6]}$ The responsibilities of each stakeholder are defined although some practical points need to be clarified. In this context, it is important to consider the off-label prescription management by taking this new mechanism into account as well as those situations that this mechanism might not cover. In September 2012, this question was dealt with at one of the workgroups of the Giens pharmacological meeting. The purpose of this article is to present the proposition of this group in order to identify, to control, to announce and to monitor the off-label prescriptions.

\section{Methods}

During the first session, the group adopted a consensual definition. Then all the situations in which the off-label prescriptions were done and the identification means have been listed.

Finally, the needed mechanisms to be implemented by the health authorities for monitoring the off-label prescriptions and for validating certain common-sense situations have been raised.

\section{1. Definition of the off-label prescription}

The group agreed on the simplest and broadest definition. The off-label prescriptions gathered all the prescriptions out of the scope defined by the summary of the product characteristics (SPC). These situations especially address the indication and dosage headings of the SPC, but also specific population for which the usage is limited, often due to a lack of exploratory data.

The need of care is clearly important in populations such as children, pregnant women, elderly patients, patients with a body mass index $>25$, patients suffering from kidney or liver failure. 
This off-label practice is widespread around 15 to $20 \%$ of all prescriptions and even higher proportion in pediatric, gerontology, cardiology, and cancer cares. ${ }^{[1]}$

\subsection{The reasons for off-label prescriptions}

The off-label prescription is necessary for specific clinical situation. Indeed, despite an increasingly wide-ranging portfolio and more and more specific therapies, there are too many clinical situations for which no therapeutic solution has been validated by MA. In such situations, the prescriber has to choose the more appropriate therapeutic solution according to limited results often insufficient for a MA.

In other situations, robust available clinical data have not yet been submitted for an extended MA. Indeed, there is frequently a delay between the knowledge enhancement and MA extension.

Usually, for the purpose of a product registration, studies are performed in strictly selected patients according to inclusion/exclusion criteria. Such studies do not provide for a risk/benefit ratio analysis in special populations such as: geriatrics or pediatrics. Nevertheless sixty per cent of reimbursed drugs are prescribed in patients older than 65 years. ${ }^{[7]}$

Off-label prescribing is a risky clinical practice. First for the patient, because benefit/risk ratio is unknown, particularly for safety reasons ; secondly for the practitioner assuming the legal responsibility, third for the pharmaceutical company accountable but not systematically informed of this off-label prescriptions and finally for the social security for which the risk becomes financial as the cost of the drug prescribed off-label will be reimbursed although the prescription is not necessary justified and for a population group that was, in many cases, not included among the initial targeted population.

\subsection{What off-label identification and follow-up systems are available?}

There are many sources for identifying off-label prescriptions but they are not yet structured. Off-label prescriptions are not easy to track because if the prescriber has to write "off-label" on his prescription, obviously, in practice, he will only do so in exceptional cases. Pharmacists who dispense the drug, as well as the social security that reimburses for it, do not know the diagnosis (or the targeted indication); therefore, in order to identify off-label prescriptions, we have to be able to share the databases.

These databases are the tracking database of pharmacovigilance, hospital monitoring drug, the sales databases or market surveys of pharma industries (sales volume versus target population), or even databases from studies in real life conditions and also data provided by the social security. However, these different sources are often independent and not yet shared. It would be necessary to pool them in order to obtain complete and representative information, but this task is today very complex. The monitoring of scientific literature, however, may highlight off-label practices from public sources. Furthermore, some off-label uses may also emerge by the media with high-profile public health problems requiring that these medical practices be examined more closely. These practices should be further analyzed. Whatever the source is, sharing prescriptions and diagnosis appear as the main concern. The computerized patient file could be a solution.

\subsection{Off-label monitoring mechanisms available in 2012}

Article R4127-8 of the public health code has recognized, since 1971 , the doctor's freedom to prescribe, which must take into account the benefits, the risks and the consequences of such prescriptions either for additional examinations or for drugs.

The statement: "off-label prescription" is thus essential and means that the cost of the drug will not be reimbursed.

Prior to the Law of December 29, 2011 (2), off-label prescriptions were controlled by means of 2 mechanisms:

1. National best practice guidelines for prescription of drugs on the off-homogeneous stay group (off-GHS) list drawn up by the health authorities (Afssaps, HAS and INCA) with the help of clinical experts following a scientific literature analysis and assessment of the risk/benefit ratio in not recognized pathological situations by the MA. When the drug is included in this list, the reimbursement by the social security is justified (e.g. expensive drugs, rare diseases or lack of therapeutic alternatives). This mechanism will expire by the end of June 2014.

2. The second mechanism is the overriding reimbursement known as Article 56, (L162-17-2-1 of the CSS) which authorizes the reimbursement of drugs or services by social security on a temporary exemption basis after assessment by the HAS and ANSM. It concerns drugs or services used outside the "basket" of reimbursable goods and services for the coverage of "long-term" or rare diseases. It may be or not associate with a request of a patient follow-up and of MA application. This mechanism is still in place for the time being.

\section{Results and proposals of the workgroup}

\subsection{Type of off-label use}

The off-label prescriptions are more or less justified according to the therapeutic need. But they are based on very various levels of proof. In table I, we propose various theoretical but pragmatic 
Table I. Types of different responses to be envisaged when an off-label prescription is detected.

\begin{tabular}{|l|l|l|}
\hline Extent of proof for favorable B/R & Need/Problem identified & Response to the need \\
\hline Existence of proof & None or poorly covered & Meets criteria for MA \\
\hline Initial proof & $\begin{array}{l}\text { None or poorly covered } \\
\text { But precautionary principle to be applied }\end{array}$ & Need to consolidate the proof \\
\hline Extrapolation of MA by common sense & $\begin{array}{l}\text { MA only in "regulated" populations } \\
\text { MA in another country }\end{array}$ & $\begin{array}{l}\text { Extrapolate MA with validity in specific populations, } \\
\text { particularly rare diseases and paediatric diseases: } \\
\text { Nommon sense MA }\end{array}$ \\
\hline $\begin{array}{l}\text { Compassionate attitude } \\
\text { Inappropriate request for drug treatment, } \\
\text { real misuse (poor use) } \\
\text { Illegal experimentation } \\
\text { Identification of new therapeutic } \\
\text { ways }\end{array}$ & Penalties \\
\hline
\end{tabular}

B/R: benefit/risk; MA: marketing autorization.

Table II. TUR: benefits and disadvantages.

\begin{tabular}{|l|l|}
\hline Benefits & Disadvantages \\
\hline Controls off-label prescriptions for a drug that already has MA & But only a portion of off-label prescriptions \\
\hline Ensures favourable B/R & Time for data collection too short (3 years) \\
\hline Enables reimbursement & $\begin{array}{l}\text { Neglects alerting bodies: manufacturers, learned societies } \\
\text { To be specified }\end{array}$ \\
\hline Expands the scope of alert & $\begin{array}{l}\text { The difficulty of claiming MA at national level based on correct extent of } \\
\text { proof data } \\
\text { Euro-compatible for European MAs }\end{array}$ \\
\hline Capacity to reduce the scope of off-label prescriptions & \begin{tabular}{l} 
Still some questions as to how the agreement will be established \\
\hline
\end{tabular} \\
\hline
\end{tabular}

B/R: benefit/risk; MA: marketing authorization; TUR: temporary use recommendations.

responses for answering to an off-label use, according to the reliability of the scientific proofs and the real medical need.

\subsection{An initial response for the control of a justified off- label prescription: temporary use recommendations and development proposals}

As defined in the drug reform of December 2011 temporary use recommendations (TUR) is a mechanism to officially manage the off-label prescriptions, justified but only under strict conditions. Medicines with at least a MA involved may be managed by this new system.

TUR are possible if there is no medically appropriate alternative with MA or a no ongoing early access program. The TURs are designed by the ANSM and are accompanied by a mandatory monitoring of effectiveness and safety data.
They are associated with an agreement signed between the ANSM and the market authorization holder(s) with a potential commitment to eventually file a further MA application. ${ }^{[8]}$

This new mechanism has both advantages and disadvantages (table II); two of these are particularly restrictive: the 3 year-period is too short for collecting data for a future MA application file and the euro compatibility of such a system has to be discussed at European level.

A third problem that could be discussed is to request to file a MA extension for a genericized product. Facilitating procedures may be implemented, such as simplified procedures for a generics manufacturer or even academic holder.

The recommendations of the workgroup for managing the offlabel usages could be different according to the level of scientific proof of the $\mathrm{B} / \mathrm{R}$ ratio and the existence or not of therapeutic alternatives (table III).

1. In the ideal scenario, the availability of scientific proofs with a favorable risk/benefit ratio must enable a MA registration dossier, 
Table III. Proposals for managing off-label prescribing.

\begin{tabular}{|l|l|l|l|}
\hline Extent of proof for favourable B/R & Need/Problem identified & Response to the need & Proposals \\
\hline Existence of proof & None or poorly covered & Meets criteria for MA & $\begin{array}{l}\text { Concomitant TUR+submission of a MA by } \\
\text { literature file. }\end{array}$ \\
\hline Initial proof & $\begin{array}{l}\text { None or poorly covered } \\
\text { precautionary principle } \\
\text { sense }\end{array}$ & $\begin{array}{l}\text { Need to consolidate the proofs } \\
\text { populations } \\
\text { MA in another country }\end{array}$ & $\begin{array}{l}\text { Clinical trials+ } \\
\text { TURs }\end{array}$ \\
\hline No proof & $\begin{array}{l}\text { Compassionate attitude } \\
\text { Inappropriate request for } \\
\text { drug treatment, } \\
\text { real misuse (poor use) } \\
\text { Illegal experimentation } \\
\text { Identification of new avenues for } \\
\text { treatment }\end{array}$ & $\begin{array}{l}\text { Start assessment } \\
\text { inseases }\end{array}$ & $\begin{array}{l}\text { Ranging from education to penalties } \\
\text { Propose a different therapeutic attitude } \\
\text { Set-up a real controlled research }\end{array}$ \\
\hline
\end{tabular}

TUR: temporary use recommendations; UR: use of recommendation.

with the concomitant 3 year TUR permitting the availability of the product up to the MA approval.

2. In many cases, the 3-year period of the TUR will not appear sufficient for obtaining reliable data in order to file an MA application. The creation of a renewable TUR would therefore allow to generate sufficient data.

3. Whether, this new period would be still insufficient and with an unmet medical need, a use recommendation (UR) could be drafted. Such as UR would address small population groups, and/ or old molecules for which their use would be validated by the appropriate learned societies by the mean recommendations and sold at the existing price (this procedure is quite different from article 56).

The URs would be accompanied by a patient follow-up with different objectives to be specified. The prescription could be performed by a specific circuit with a dedicated prescription order. This UR could be reassessed every 5 years. As with the TURs, this mechanism naturally cannot be effective and reliable without appropriate communication to the appropriate prescribers, pharmacists, and patients (figure 1).

If the data have been collected for a general population and have led to a MA in a sufficiently broad population, mathematical models may be used to extrapolate pharmacokinetic data in more restricted population groups and a "common-sense" use recommendation should be issued and would monitor such as off-label prescription without the paperwork of a new MA application.

4. Finally, in the absence of real clinical proofs, either misuse must be prohibited (some actions have to be implemented in this aim from education to penalties), or a clinical development has to be initiated for having a real assessment of the risk/benefit ratio, particularly in public health domains, with a lack of public alternative launched via public funding (like clinical research program [programme hospitalier de recherche clinique, $\mathrm{PHRC}$ ] or other calls for tender).

\subsection{Need to supervise and control all off-label prescriptions: an off-label committee}

As we have just seen with the TURs, the ANSM has adopted an effective mechanism for controlling medically justified off-label use supported by relatively solid data. This mechanism, however, has its limits and will not be able to control all off-label prescriptions. An off-label committee may be created to cover all off-label situations. It would be composed of representatives from the HAS, the regional pharmacovigilance centers (Centre régional de pharmacovigilance, CRPV), the TUR unit of the ANSM, the Directorate 


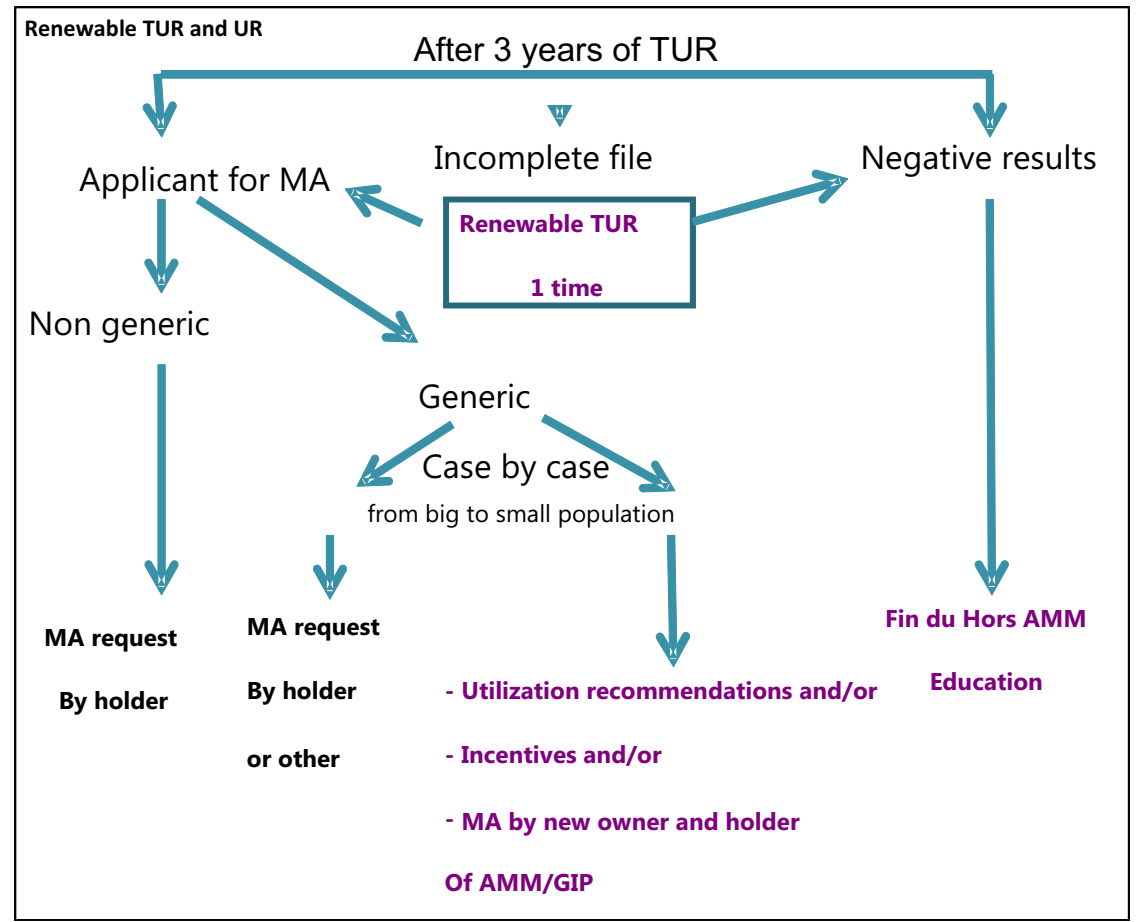

Fig. 1. Proposed circuit of TUR and UR.

GIP: public interest group (GIP-CenGEPS); MA: marketing authorization; TUR: temporary use recommendations; UR: use of recommendation.

General for Health (Direction générale de la santé, DGS), the Directorate for Social Security (Direction de la sécurité sociale, DSS), and the National Health Insurance Fund (Caisse nationale d'Assurance maladie, CNAM).

It would perform the following missions:

- monitoring of off-label prescriptions by consulting and analyzing existing databases and monitoring of international scientific literature;

- drafting of pharmaco-epidemiological-economic protocols;

- research into mathematical models on population pharmacokinetics for risk population, such as the elderly patients or pregnant women;

- implementation and monitoring of TURs;

- implementation of mechanisms to monitor the off-label prescriptions in public health indications (such as the CAMTEA, protocol implemented in the north of France for controlling the use of lioresal in alcoholic dependence);

- realization of an old drugs overview by assessing their benefit/risk ratio with any support from reference centers (generics and old drugs with very broad MAs without a welldefined indication);

- education programs and communication towards the various health actors through information letters to healthcare professionals, web publications, off-label newsletters, etc.;

This committee may also make recommendations according to different identified situations in order, ultimately, to encourage the "MA track" according to therapeutic need.

\section{Conclusion}

Following serious reflection with the participation of actors from many different fields, three important proposals have been made by the group:

1. The possibility of a TUR that can be renewed once or up to a maximum of six years.

2. The possibility of obtaining a Use Recommendation (UR), for certain drugs in very specific situations, that can be renewed every five years in the absence of an alternative and a sponsor to lead the project as long as tolerance is not problematic.

3. And particularly, with a view to control all these mechanisms, the creation of a joint off-label committee that would supervise off-label prescriptions in everybody's interest and mainly the patient's and sometimes grant so-called common-sense MAs, particularly for restricted population groups.

Conflicts of interests. None.

Abbreviations. Afssaps: French Agency for the Safety and Health Products (Agence française de sécurité sanitaire des produits de 
santé); ANSM : National Security Agency of Medicines and Health Products (Agence nationale de sécurité du médicament et des produits de santé); CEPS: Economic Committee for Health Products (Comité économique des produits de santé); EGB: general sample of beneficiaries (échantillon généraliste de bénéficiaires); HAS : High Health Authority (Haute autorité de santé); INCA: National Institute against Cancer (Institut national contre le cancer); MA: marketing authorization; PMSI: medicalized information system program (programme de médicalisation des systèmes d'information); PTT: temporary treatment protocol (protocole thérapeutique temporaire); SNIIRAM: national inter-regional Health insurance information system (système national d'informations inter-régions d'Assurance maladie); SPC: summary of product characteristics; TUR: temporary use recommendation

\section{References}

1. Rapport de synthèse des Assises du médicament. Groupe 3. http://www.legifrance.gouv.fr

2. Article 56 de la loi de financement de la sécurité sociale. http://www.legifrance.gouv.fr
3. Loi n 2011-2012 du 29 décembre 2011 relative au renforcement de la sécurité sanitaire du médicament et des produits de santé

http://www.legifrance.gouv.fr

4. Décret $\mathrm{n}^{\circ}$ 2012-597 du 27 avril 2012 relatif à l'Agence nationale de sécurité du médicament et des produits de santé. http://www.legifrance.gouv.fr

5. Décret $n^{\circ}$ 2012-742 du 9 mai 2012 relatif aux recommandations temporaires d'utilisation des spécialités pharmaceutiques. http://www.legifrance.gouv.fr

6. Décret $\mathrm{n}^{\circ}$ 2012-740 du 9 mai 2012 relatif à la prise en charge dérogatoire par l'assurance maladie des spécialités pharmaceutiques bénéficiant d'une recommandation temporaire d'utilisation ou de certains produits et prestations. http://www.legifrance.gouv.fr

7. Laude A. Dans la tourmente du Mediator : prescription hors-AMM et responsabilités. Recueil Dalloz, 27 janvier 2011, n4

8. Emmerich J, Dumarcet N, Lorence A. France's new framework for regulating off-label drug use. N Engl J Med 2012 Oct 4; 367(14): 1279-81
Correspondence and offprints: Claire Le Jeunne, Service de Médecine interne, Assistance Publique - Hôpitaux de Paris, Hôtel Dieu, 1 place du Parvis de Notre Dame, 75004 Paris, France.

E-mail: claire.le-jeunne@htd.aphp.fr 